\title{
Comparison of the static in vivo approach to a physiologically based pharmacokinetic approach for metabolic drug-drug interactions prediction
}

\begin{abstract}
Background: The in vivo mechanistic static model (IMSM) and the physiologically based pharmacokinetic (PBPK) model are two approaches used to predict the magnitude of drug-drug interactions (DDIs). The aim of this study was to evaluate the performance of IMSM and to compare IMSM with the PBPK approach implemented in Simcyp. Methods: The predictive performances of IMSM were evaluated on a panel of 628 DDIs. Subsequently, the IMSM and PBPK approaches were compared on a set of 104 DDIs. Results: The IMSM yielded $85 \%$ of predictions within 1.5 -fold of the observed value on the $628 \mathrm{DDIs}$ panel. The predictive performances of IMSM were better than those of the PBPK approach (median fold error 1 vs 0.86 on 104 studies; $p=0.02$ ). Conclusion: The IMSM approach is an alternative tool for metabolic DDIs prediction.
\end{abstract}

First draft submitted: 20th November 2015; Accepted for publication: 2nd February 2016; Published online: 4 April 2016

Keywords: cytochromes $\bullet$ drug-drug interaction $\bullet$ evaluation $\bullet$ mechanistic model - prediction

Pharmacokinetic drug-drug interactions (DDIs) are an important issue because they may alter the efficacy and the safety of many drugs. Among the mechanisms leading to clinically relevant DDIs, interactions on conjugation enzymes (e.g., glucuronidation [1]) are quite scarce, while metabolic interactions on cytochrome P450 (CYP) enzymes are very frequently involved. To cite just one example, the exposure to midazolam may vary 400 -fold depending on whether it is associated with rifampicin (a potent inducer of CYP3A4) or itraconazole (a potent inhibitor of CYP3A4) [2]. Therefore, the health authorities require that the potential for DDIs of a drug candidate be evaluated before approval $[3,4]$. The magnitude of an interaction is usually expressed as the ratio of the victim drug AUC given in combination with an interacting drug (i.e., inducer or inhibitor) to the victim drug AUC given alone. Quantitative prediction of the magnitude of a DDI is useful to identify the clinical interaction studies to be performed in drug development, and the dosing adaptation to be made in the context of drug prescription. Three main kinds of approaches for quantitative predictions of metabolic DDIs have been described. They will be presented here by historical order of appearance. The first approach rely on in vitro determination of the type of inhibition (competitive, noncompetitive or suicide), and measurements of the characteristics of substrates and inhibitors. Coupled with a mechanistic static interaction model (MSM), the predictive performances of this method were encouraging, approximately $80-85 \%$ of predicted exposures within twofold of in vivo value [5-8]. The second approach relies on the same in vitro data, plugged into a physiologically based pharmacokinetic (PBPK) model $[9,10]$. This approach, also called the mechanistic dynamic interaction model (MDM), demonstrated better predictive performances
Michel Tod ${ }^{*}, 1,2$, Philippe

B. Pierrillas ${ }^{2}$, Laurent Bourguignon ${ }^{3,4}$ \& Sylvain Goutelle 3,4

'Pharmacie, Hôpital de la Croix-Rousse, Hospices Civils de Lyon, Lyon, France ${ }^{2}$ EMR 3738, Faculté de médecine Lyonsud, BP 12, Chemin du grand revoyet, 69921 Oullins, Université Lyon 1, Lyon, France

3Pharmacie, GHG, Hospices Civils de Lyon, Lyon, France 4UMR CNRS5558, Laboratoire de Biométrie et Biologie Evolutive, Université Lyon 1, Lyon, France

*Author for correspondence:

Tel.: +33472072663

Fax: +33472071894

michel.tod@chu-lyon.fr 
than the former [11]. The last approach rely on in vivo data only, coupled with a mechanistic static interaction model, and will be denoted in vivo mechanistic static model (IMSM). It was introduced by Ohno [12,13] and extended in several directions by our group [14-19]. It demonstrated good predictive performances on moderate size studies $(\mathrm{n}=50-100)$ devoted to DDIs mediated by each of the main CYPs. However, to date, the IMSM approach has not been evaluated on a large panel of DDIs and has not been compared with a PBPK approach. The current study was undertaken to fill these gaps.

\section{Methods}

The prediction models

A rigorous and quite exhaustive derivation of the equations involved in the static in vivo approach for metabolic DDIs prediction has been presented by Hisaka et al. [20]. For clarity, the main equations and assumptions associated with the three approaches are summarized here. For simplicity, but without loss of generality, the victim drug is assumed to be eliminated by a single cytochrome in the liver, and also by other hepatic mechanisms (metabolism or biliary excretion), and possibly by renal route. Consideration of elimination by several CYPs would simply involve summation terms.

The fraction of elimination clearance (CL) due to cytochrome metabolism is denoted as CR (contribution ratio), defined as:

$$
C R=\frac{(C L / F)-\left(C L_{-C Y P} / F_{-C Y P}\right)}{\left(C_{L} / F\right)}
$$

where $\mathrm{CL}_{-\mathrm{CYP}}$ and $\mathrm{F}_{-\mathrm{CYP}}$ are the clearance and the bioavailability of the victim drug when the cytochrome activity is suppressed, for example, by mutation on both CYP alleles or complete inhibition by an inhibitor. CR may be calculated after intravenous or oral administration, and the corresponding values may differ. Note that CR is defined with respect to total clearance, not hepatic clearance.

A general equation to calculate the AUC ratio in case of metabolism inhibition, irrespective of administration route, is [21]:

$$
\frac{A U C^{*}}{A U C}=\frac{C L / F}{C L^{*} / F^{*}}=\frac{F^{*}}{F} \cdot \frac{1}{\frac{C R}{1+B}+(1-C R)}
$$

where $B=\frac{I_{h, u}}{K_{i}}$

is used for competitive or noncompetitive inhibition, and

$$
B=\frac{k_{\text {inacc }} h_{h, u}}{K_{\text {deg }}\left(h_{h, u}+K_{i}\right)}
$$

is used for mechanism-based inhibition.

$\mathrm{I}_{\mathrm{h}, \mathrm{u}}$ is the intracellular unbound concentration of the inhibitor in the liver, $\mathrm{K}_{\mathrm{i}}$ is an inhibition constant, $\mathrm{k}_{\mathrm{in}-}$ act and $\mathrm{k}_{\mathrm{deg}}$ are rate constants and $\mathrm{K}_{\mathrm{I}}$ is the concentration of inhibitor when the rate constant of inactivation reaches half kinact. The superscript * denotes a parameter value when the victim and inhibitor drugs are combined. The assumptions on which these equations rely are the following:

- The metabolic pathway follows a Michaelis-Menten (MM)-type kinetics.

- The intracellular substrate concentration $\mathrm{C}_{\mathrm{u}}$ is well below the Michaelis constant, $\mathrm{Km}$ (in other words, $\mathrm{CL}$ is independent of substrate dose).

- The penetration of the substrate into liver cells is perfusion-limited (diffusion is rapid).

- Unbound concentrations of the substrate in liver cell water and plasma water are equal (no active transport, and bound drug does not cross membranes).

- $\mathrm{I}_{\mathrm{h}, \mathrm{u}}$ is constant (approximately equal to the timeaverage concentration of unbound inhibitor).

Note that the AUC ratio is always lower after intravenous administration of the victim drug or the inhibitor than after oral administration, because CR and $\mathrm{I}_{\mathrm{h}, \mathrm{u}} / \mathrm{K}_{\mathrm{i}}$ are lower. Hence, efforts to predict DDIs have been primarily devoted to oral administration.

In case of oral administration of the victim drug and the inhibitor:

$$
\frac{A \cup C^{*}}{A \cup C}=\frac{f u \cdot C L i / F_{g}}{f u^{*} \cdot C L i^{*} / F_{g}{ }^{*}}=\frac{F_{g}{ }^{*}}{F_{g}} \cdot \frac{1}{\frac{C R}{1+B}+(1-C R)}
$$

where CLi is the hepatic intrinsic clearance of the victim drug, $\mathrm{fu}$ is the unbound drug fraction and $\mathrm{F}_{\mathrm{g}}$ is the fraction of drug escaping from the intestine. Additional assumptions required by this equation are:

- Hepatic clearance is described by the well-stirred mode;

- Elimination clearance is equal to hepatic clearance: renal clearance is assumed to be null;

- The fraction of the substrate drug absorbed $\left(\mathrm{F}_{\mathrm{a}}\right)$ is assumed to be constant (independent of the inhibitor);

- Substrate fu is assumed to be constant (no displacement by the inhibitor: $\mathrm{fu} / \mathrm{fu}^{*}=1$ );

- Substrate blood to plasma concentration ratio is assumed to be constant (independent of the inhibitor). 
The in vitro approach, also known as mechanistic static model

The parameters $\mathrm{B}$ and $\mathrm{CR}$ are estimated from in vitro data. The value of CR is approximated by $\mathrm{fm}$, the fraction of hepatic intrinsic clearance (not elimination clearance) due to the cytochrome pathway. When the renal clearance is not negligible and $\mathrm{F}_{\mathrm{g}}=\mathrm{F}_{\mathrm{g}}{ }^{*}$, Hisaka [20] showed that the relationship between $\mathrm{fm}$ and $\mathrm{CR}$ is:

$$
C R=\frac{f m}{1+\frac{1}{f u \cdot C L i\left(\frac{1}{C L_{r}}+\frac{1}{B P R \cdot Q_{h}}\right)}}
$$

where $\mathrm{CL}_{\mathrm{r}}$ is the renal clearance of the victim drug, $\mathrm{Q}_{\mathrm{h}}$ is liver blood flow and BPR is the blood to plasma concentration ratio of the drug. When $\mathrm{CL}_{\mathrm{r}}=0, \mathrm{CR}$ and $\mathrm{fm}$ are equal.

The calculation of $\mathrm{I}_{\mathrm{h}, \mathrm{u}}$ to be plugged in Equation 2 is a matter of debate. In the US FDA guidance [4], this value is calculated as follows:

$$
I_{h, u}=f u_{b} \cdot\left[I_{\max , b}+\frac{k_{a} \cdot F_{a} F_{g} \cdot D}{Q_{h}}\right]
$$

where all parameters pertain to the inhibitor. Here, the assumptions are:

- The mechanism of inhibition has been correctly identified (mechanism-based or other);

- $\mathrm{Ki}$ is independent of the substrate;

- $\quad \mathrm{B}$ and fm have the same value in vitro and in vivo;

- The inhibitor is not metabolized, or the metabolites have no impact on the interaction;

- Penetration of the inhibitor into liver cells is perfusion-limited (diffusion is rapid);

- Unbound concentration of the inhibitor in liver cell water and plasma water are equal (no active transport, and bound drug does not cross membranes). However, a partition coefficient $\mathrm{Kp}=\mathrm{I}_{\mathrm{h}, \mathrm{u}} / \mathrm{I}_{\mathrm{p}, \mathrm{u}}$ may be used if this condition is not satisfied.

The in vivo approach, also known as in vivo mechanistic static model

This approach assumes that the AUC ratio for a DDI caused by a CYP inhibitor is as follows:

$$
\frac{A U C^{*}}{A U C}=\frac{F_{g}^{*}}{F_{g}} \cdot \frac{1}{1-C R . I R}
$$

where IR, the inhibition ratio, is a characteristic of the inhibitor for a given CYP. By comparison with Equation 2, Equation 6 implies:

$$
I R=1-\frac{C L^{*} / F^{*}}{C L / F}=\frac{B}{1+B}
$$

Note that IR is defined with respect to the elimination clearance, not to hepatic clearance. Assuming that $\mathrm{F}_{\mathrm{g}}{ }^{*} / \mathrm{F}_{\mathrm{g}}=1$, both $\mathrm{CR}$ and $\mathrm{IR}$ are calculated from in vivo data. The CR parameter may be calculated as follows:

$$
C R=\frac{(\text { AUC*/AUC })-1}{(\text { AUC*/AUC }) \cdot I R}
$$

using the AUC ratio of the victim drug whose CR is to be calculated, obtained with an inhibitor whose IR is known. The calculation of IR is:

$$
I R=\frac{\left(A \cup C^{*} / A \cup C\right)-1}{\left(A \cup C^{*} / A \cup C\right) \cdot C R}
$$

using the AUC ratio of a victim drug whose CR is known, obtained with an inhibitor whose IR is to be calculated.

The assumptions behind these equations are:

- IR is independent of the substrate. This has been thoroughly evaluated for CYP3A4 inhibitors [19];

- IR depends on the dose (actually the concentration) of the inhibitor, because in Equation 7, B depends on $\mathrm{I}_{\mathrm{h}, \mathrm{u}}$;

- As stated above, $\mathrm{fu}^{*} / \mathrm{fu}=1$ and $\mathrm{F}_{\mathrm{g}}{ }^{*} / \mathrm{F}_{\mathrm{g}}=1$. The validity of the first assumption is not an issue: if $\mathrm{fu}^{*} / \mathrm{fu}$ is not equal to 1 , the calculation from EQUATION 6 yields in fact (fu*.AUC*)/(fu.AUC), that is, the ratio of unbound AUCs, which is actually the measure of interest for drug-dose adjustment. The second assumption may be an issue for CYP3A4 only, because the other CYPs have negligible activity in the gut, so $\mathrm{F}_{\mathrm{g}}$ and $\mathrm{F}_{\mathrm{g}}^{*}$ are close to one. With CYP3A4, as shown earlier by simulation [20] and by studying DDIs with the rather selective inhibitor grapefruit juice [19], the lack of consideration of the variation of $\mathrm{F}_{\mathrm{g}}$ has been shown to have little impact on the predictive performances.

\section{The PBPK approach, also designated as mechanistic dynamic model}

$\mathrm{I}_{\mathrm{h}, \mathrm{u}}$ is not treated as constant, that is, the inhibitor concentration versus time $(t)$ profile $I_{h, u}(t)$ is properly taken into account. Depending on the complexity of the PBPK model, all other assumptions may be relaxed or not.

Although the methods have been presented here in the context of inhibition, description of interactions by induction is also possible with similar concepts [12,22]. In this case, the potency of the inducer is described by a parameter named inductive capacity (IC). These methods may be extended in several directions: 
- In case of a DDI involving multiple CYPs, by 'summing' the contributions of each pathway using the respective CRs, IRs and ICs (or their equivalent) [16],

- In case of CYP polymorphism, by modifying the CRs by a factor characterizing the impact of the variant genotype relative to the wild genotype $[15,16]$,

- In case of cirrhosis, mainly by reducing the CRs by a factor depending on the severity of the disease, according to the Child-Pugh score [23].

The MSM approach has been compared with the MDM approach in a number of papers [11,24,25]. As a result, although the MSM approach works quite good, the MDM approach is generally considered to perform better. This is why we considered MDM as the gold standard, and chose to compare IMSM to MDM.

\section{Evaluation of the IMSM predictions}

The IMSM approach is currently implemented in the website www.ddi-predictor.org. The website returns predicted AUC ratios for DDIs (171 substrates, 94 inhibitors and 21 inducers) mediated by CYPs $1 \mathrm{~A} 2$, 2D6, 2C9, 2C19 and 3A4 [14-19], allowing $171 \times(94$ $+21)=19,665$ DDIs to be predicted. Multiple interactions between two drugs, involving several CYPs or simultaneous inhibition and induction, are also accommodated. These drug interactions may also be predicted according to the genotype of the patient, in case of polymorphic CYPs (i.e., 2D6, 2C9 and 2C19) $[15,17,18]$, and in case of cirrhosis, according to Child-Pugh severity score [23]. The website includes a database for validation purpose: predictions for a large number of substrates-interactor pairs may be compared with published data. For this article, all the published AUC ratios of the DDI database were extracted, and compared with the predicted AUC ratios, obtained by the full equation implemented in the website [16]:

$$
\frac{A U C^{*}}{A \cup C}=\frac{1}{\sum_{i} C R_{i}\left(1+I X_{i}\right)+\left(1-\sum_{i} C R_{i}\right)}
$$

where the summation is over all the CYPs known to be involved in the metabolism of the drug, and IX is the potency of the inhibitor or the inducer. If the interacting drug is an inhibitor for the $\mathrm{i}$-th CYP, then IX = IR and its value is negative. If the interacting drug is an inducer for the $\mathrm{i}$-th CYP, then IX = IC and its value is positive.

To allow comparison with previous publications, the same metrics of performances were used. The bias was assessed by the mean fold prediction error of the AUC ratio:

$$
M F E=10^{\frac{1}{n}} \sum\left|\log _{10}\left(\frac{\text { Rpred }}{\text { Robs }}\right)\right|
$$

where $\mathrm{n}$ is the number of studies and Rpred, Robs are the predicted and observed AUC ratios, respectively.

The imprecision was assessed by the root meansquared error:

$$
R M S E=\sqrt{\frac{1}{n} \sum(\text { Rpred }- \text { Robs })^{2}}
$$

The proportions of predictions within twofold and 1.5-fold of the observed AUC ratio were computed.

More robust metrics of performances were also calculated: the median (MDFE) and the interquartile range (IQFE) of fold errors. The standard errors of the mean fold error and MDFE were computed by bootstrap (100 replications). The MDFEs were compared by the Wilcoxon signed-rank test.

\section{Comparison of the IMSM \& MDM predictions}

Two large datasets dealing with the evaluation of the MDM approach implemented in the Simcyp Population-Based ADME Simulator (Simcyp Ltd., Sheffield, UK) have been published previously [11,25]. We extracted the observed and predicted AUC ratios from these two publications. The corresponding predictions provided by the IMSM approach were calculated using Eguation 9 implemented in www.ddi-predictor.org. The average fold error (AFE), root mean square error, MDFE and IQFE were computed for each method.

\section{Results}

\section{Evaluation of the IMSM predictions}

The analysis of the database of published interactions in ddi-predictor website retrieved 628 predictions of metabolic interactions. These predictions covered 142 substrates combined with at least one of 95 interactors (18 inducers, 77 inhibitors). The plot of the predicted versus observed AUC ratios for the 628 predictions is shown in Figure 1A. There were 30 values $(4.8 \%)$ outside the $50-200 \%$ interval around the observed value. There was no clear explanation for these discrepancies. The distribution of fold errors is shown in Figure 1B. The predictive performances of the MDM and IMSM approaches are presented in Table 1. In this table, the performances reported for the MDM approach were taken from the original publications [11,24,25], and were computed on a set of studies different from ours. Hence the results are not directly comparable. The MDFE and IQFE of IMSM were 1 and 0.27 , respectively.

\section{Comparison of the IMSM \& MDM predictions}

To afford a better, unbiased comparison between the IMSM and MDM approaches, a dataset allowing a 
direct comparison was built, starting from Einolf's dataset [11], which originally contained 100 interaction studies. Among these, one study with mephenytoin was discarded because the dose of fluvoxamine was lower than that computed in our IMSM approach. One midazolam-itraconazole study was discarded because itraconazole was stopped 4 days before midazolam administration. Fifteen other studies were discarded because they involved a single dose of an inhibitor known to have a long half-life, leading to some degree of accumulation upon repeated dosing, which can affect the IR value (IR is greater after repeated dosing than after single dose in this case). Since the IRs were calculated after repeated administration, these data were not suitable for the comparison. The remaining studies were merged with the data reported by Guest [25] for interactions between benzodiazepines and azole antifungal agents (seven studies), and recent data from the literature, allowing to add fourteen studies. Finally, 104 studies could be included in the direct comparison (Supplementary Table 1). These 104 studies involved 21 substrates and 18 inhibitors. The predictive performances of the MDM and IMSM approaches are compared in Table 2 and in Figures $2 \& 3$.

The MDFE $(0.86 \pm 0.036)$ was significantly different from unity for the MDM approach $(\mathrm{p}<0.01)$, but not for the IMSM approach $(1 \pm 0.024)$. The MDFE of IMSM and MDM were significantly different ( $\mathrm{p}=$ 0.02 by the Wicoxon signed-rank test).

\section{Discussion}

In this study, two kinds of results are presented. First, the largest evaluation reported so far of the IMSM for quantitative prediction of CYP-mediated DDIs is presented. Second, this approach is compared with the MDM for quantitative prediction of the AUC ratio of DDIs in a subset of data.

Regarding the first evaluation, carried out on 628 studies, the performance metrics show that the IMSM approach works very well, at least on these examples. Arguably, the database of DDI-predictor website contains only the CYP substrate drugs and interactors that are deemed to accommodate to this approach. The IMSM approach works correctly if cytochromes are the main interaction mechanism, and the kinetics of the substrate is (at least approximately) linear. When the main mechanism of interaction is different, for example, competition on a transporter, the current IMSM approach is likely to fail. Strong interactions of this type have been described for a limited number of drugs such as statins or glinides when coadministered with cyclosporin $[26,27]$. Other examples of interactions with a complex mechanism that were successfully described by a PBPK model are repaglinide-gemfibrozil [28], repaglinide-rifampicin [29], interactions with amiodarone and its active metabolite [30], and midazolam-AZD2327 [31]. Apart from this issue, the IMSM approach is robust, in the sense that it is not fooled by erroneous in vitro data (which are not used) or by complexities such as metabolites with inhibiting properties. There are examples of important discrepancies between in vitro and in vivo data on drug interactions. Diclofenac has been presented as a substrate metabolized primarily by CYP2C9, based on in vitro data. Yet, in vivo, mutations on CYP2C9 that are known to impair this enzyme do not increase the exposure to diclofenac, showing that CYP2C9 is a minor pathway for diclofenac elimination [32]. Montelukast has been presented as a strong inhibitor of $\mathrm{CYP} 2 \mathrm{C} 8$ in vitro that should

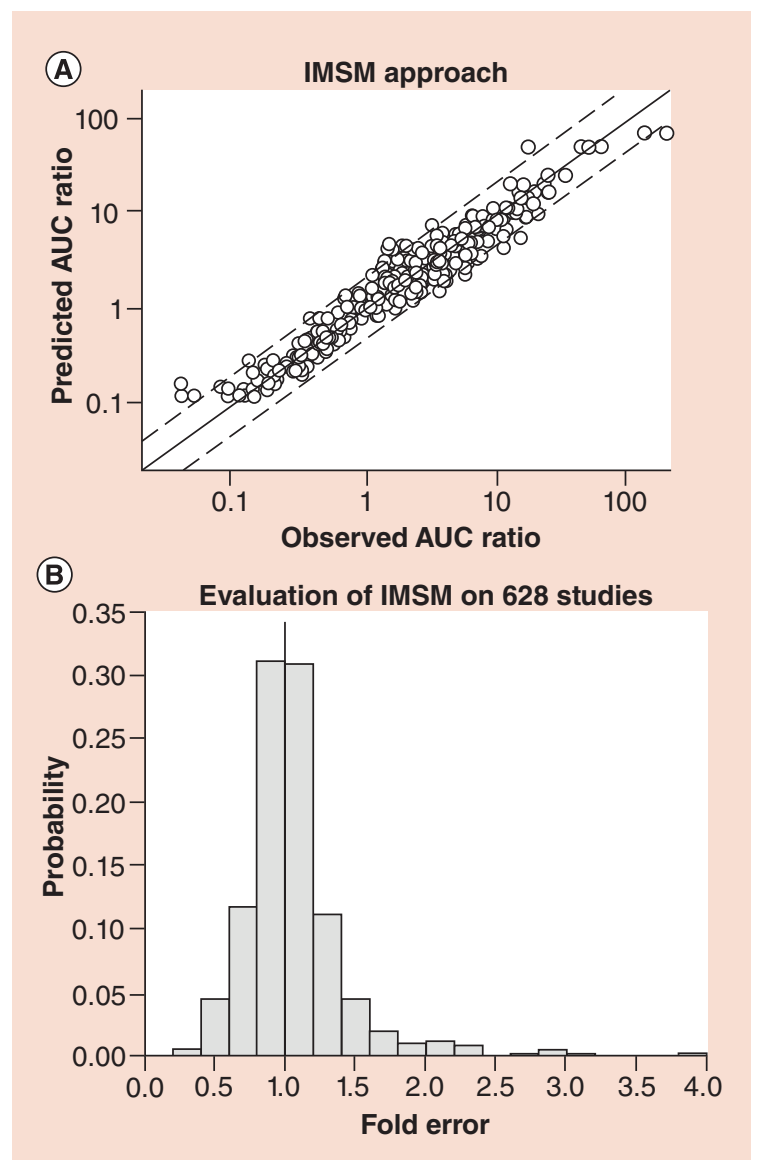

Figure 1. Evaluation of the in vivo mechanistic static model approach based on 628 published interaction studies. (A) Predicted versus observed AUC ratios. The line is the $y=x$ line. The dashed lines represent the $50-200 \%$ interval. Values above $x=1$ represent drug-drug interactions (DDIs) by inhibition. Values below $x=1$ represents DDIs by induction. (B) Histogram of the fold errors (= predicted/observed AUC ratio). The vertical line at $x=1$ represents the ideal value (no prediction bias). 
Table 1. Predictive performances of the mechanistic dynamic model (published data) and in vivo mechanistic static model approaches.

\begin{tabular}{|c|c|c|c|c|c|}
\hline & $\begin{array}{l}\text { Number and percentage of } \\
\text { predictions within twofold } \\
\text { of observed AUC ratio }\end{array}$ & $\begin{array}{l}\text { Number and percentage of } \\
\text { predictions within } 1.5 \text {-fold } \\
\text { of observed AUC ratio }\end{array}$ & MFE & RMSE & Ref. \\
\hline IMSM $(n=628)$ & $598(95 \%)$ & $534(85 \%)$ & 1.22 & $\begin{array}{l}5.65 \\
1.48 \text { * }\end{array}$ & $\begin{array}{l}\text { Own } \\
\text { data }\end{array}$ \\
\hline $\operatorname{MDM}(n=100)$ & $75(75 \%)$ & Not reported & 1.67 & 4.34 & [11] \\
\hline MDM $(n=59)$ for DDls with observed $R>2$ & $38(64 \%)$ & Not reported & 1.91 & 5.55 & [11] \\
\hline $\operatorname{MDM}(n=35)$ & $25(71 \%)$ & $15(43 \%)$ & 1.68 & 3.81 & [25] \\
\hline $\operatorname{MDM}(n=48)^{\ddagger}$ & $43(90 \%)$ & Not reported & 1.47 & 3.03 & [24] \\
\hline \multicolumn{6}{|c|}{$\begin{array}{l}\text { "Performance with three values excluded: melatonin-fluvoxamine, ramelteon-fluvoxamine } \times 2 \text {. } \\
\text { "The victim drug was midazolam in all } 48 \text { cases. } \\
\text { DDI: Drug-drug interaction; IMSM: In vivo mechanistic static model; MDM: Mechanistic dynamic model; MFE: Mean fold error; R: AUC ratio; RMSE: Root mean } \\
\text { square error. }\end{array}$} \\
\hline
\end{tabular}

be relevant in vivo, even after accounting for its high protein binding. Yet, it has shown no inhibiting properties in vivo [33]. Paroxetine is known to be an inhibitor of CYP2D6. Yet, the predicted AUC ratios from in vitro data are largely underpredicted if microsomal binding and mechanism-based inhibition are not taken into account [34]. Examples of inhibitors with stronger inhibiting properties in vivo than in vitro due to metabolites are bupropion toward CYP2D6 [35] and gemfibrozil toward CYP2C8 [36]. In these situations, the IMSM approach works better because it relies only on in vivo data to establish the CRs and IRs, and it requires only minimal assumptions about the mechanism of the interaction (the type of inhibition is not a strong determinant).
The choice of the performance metrics merits discussion. Classification of an AUC ratio prediction as 'good' when it falls within 1.5- or 2-fold around the observed value may be considered as very indulgent. This criterion has been used traditionally to evaluate PBPK models, especially in the context of interspecies scaling. But the criterion for bioequivalence is much more stringent (1.25-fold) and is used as an initial cutoff value to decide if a potential DDI deserves a clinical trial [4]. On the other hand, it may be a nonsense to desire a high accuracy of any prediction method of the mean AUC ratios. Indeed, the between-study variability of the AUC ratio for a given substrate-inhibitor pair is very large. For example, eight studies reported the interaction between midazolam and ketoconazole

Table 2. Predictive performances of the mechanistic dynamic model and in vivo mechanistic static model approaches on the same dataset.

Number and percentage of
predictions within twofold of
observed AUC ratio

All DDIs $(n=104)$

IMSM $90(87 \%)$

MDM

Number and percentage of predictions within 1.5 -fold of observed AUC ratio

MFE (SE)/MDFE (SE)

RMSE/IQFE

DDIs with observed AUC ratio $>2(n=59)$

IMSM

$70(67 \%)$

$51(49 \%)^{\ddagger}$

$1.27(0.034) / 1(0.024)$

$1.75 / 0.40$

$1.62(0.092) / 0.86(0.036)$

$3.40 / 0.52$

$3.02^{\S} / 0.52$

MDM

1.30/1

$1.85 / 0.71$

\section{$2.28 / 0.48$}

$4.35 / 0.44$

$3.83^{\S} / 0.44$

"Comparison with MSM: $p=0.14$ (chi-square test).

${ }^{\ddagger}$ Comparison with MSM: $p=0.008$ (chi-square test).

sExcluding one value (the midazolam-verapamil interaction).

DDI: Drug-drug interaction; IMSM: In vivo mechanistic static model; IQFE: Interquartile fold error; MDFE: Median fold error; MDM: Mechanistic dynamic model; MFE: Mean fold error; RMSE: Root mean square error; SE: Standard error. 
(summarized in [25]). The AUC ratio ranged from 5 to 16. Even after accounting for the differences in ketoconazole dose $(200$ or $400 \mathrm{mg}$ ) or interval between midazolam and ketoconazole intake, the variability of the AUC ratio between studies remains large. The typical standard deviation of an AUC ratio of 1,10 or 20 was estimated to $1,3.16$ or 4.5 , respectively, in our former study [19].

Regarding the second evaluation (the comparison with the MDM approach), the IMSM provided performances similar if not better than those of the PBPK approach. This result raises questions about the position of the PBPK approach as the gold standard for predicting DDIs, if the goal is mainly to estimate the AUC ratio. Of course, the IMSM approach is limited to this aspect, whereas the PBPK approach yields more information, such as the concentration profile of the victim drug in blood and tissues. Depending on the goal of the analysis, prediction of the AUC ratio may be sufficient or not. In the context of drug prescription (by a physician) or prescription analysis (by a pharmacist), the AUC ratio is enough to take decisions such as adapting the dose or choosing another drug with less interaction potential. In the context of a clinical trial, the AUC ratio may also be enough to decide which comedications are allowed or not during the trial. In the context of drug development of a new chemical entity, the AUC ratio may also be enough to decide which DDI studies have to be undertaken (see below). The PBPK approach is valuable when the mechanism of interaction is not restricted to the CYPs, or when there are several active metabolites of interest, or when assessment of the interaction into a certain tissue (e.g., brain) is essential for drug action or toxicity. To facilitate the modeling of active transport processes, an approach based on tissue-specific mRNA expression data has been described and is currently available in the PBPK software PKSim [37]. Another advantage of the PBPK approach is that the distribution of the AUC ratio may be obtained by population simulation, although the results of this kind of simulation must be regarded with caution because it is correct only if the statistical model is correct. The issue is how to account properly for the correlations between the physiological or pharmacokinetic parameters of the PBPK model. Ignoring the correlations may lead to overestimate interindividual variability.

The PBPK approach is able to cope with a wide range of conditions regarding the prediction of DDIs. Factors of variability such as CYP polymorphisms and cirrhosis may also be incorporated in the IMSM approach [15,16,23]. In the DDI-predictor website, both are accommodated. Quantitative prediction of DDIs in neonates can be done by incorporating the maturation functions of the CYPs [38] into the model. Currently, this possibility has not been implemented in the IMSM approach because there is a paucity of published data to evaluate the model.

The reasons for the quite disappointing performances of the PBPK approach needs discussion. On the one hand, the PBPK approach relies on a strong

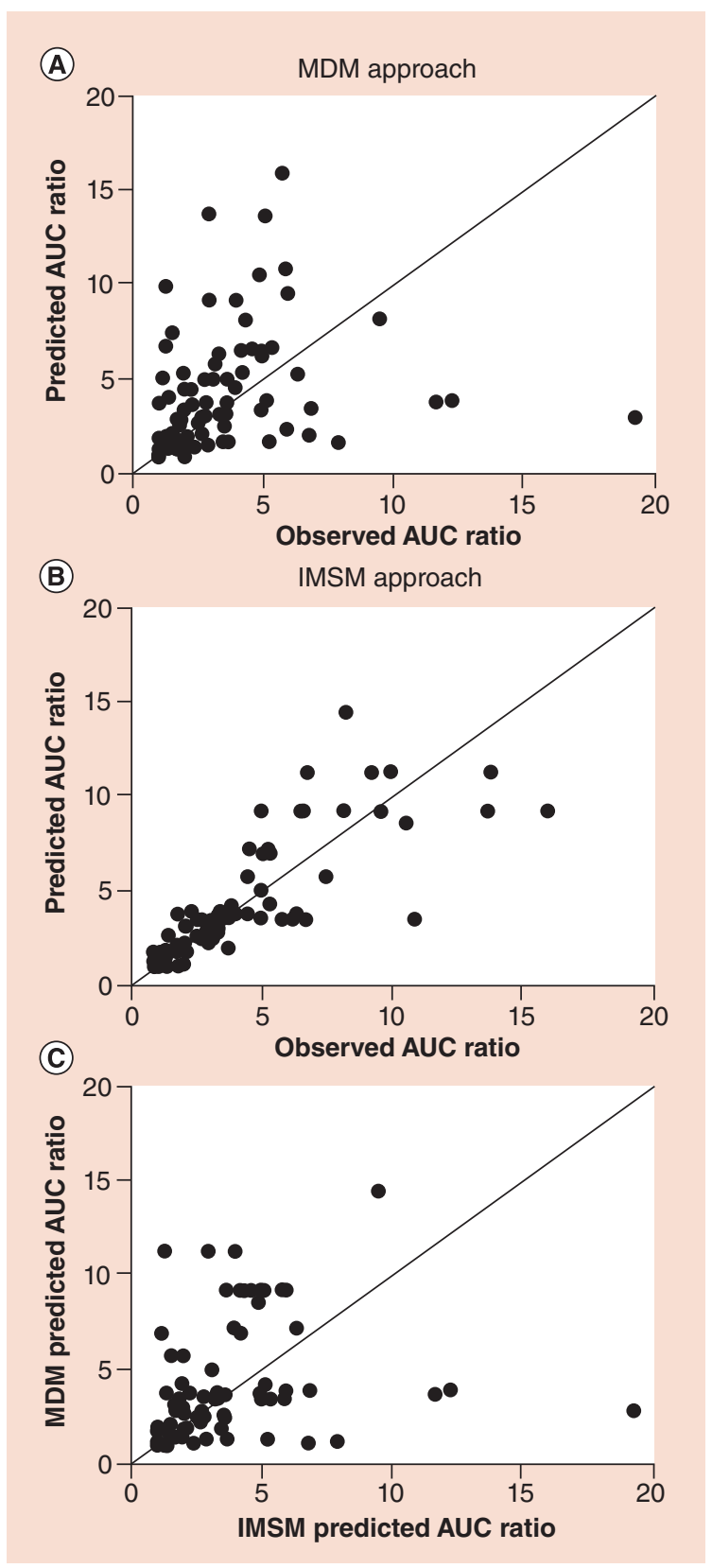

Figure 2. Comparison of the mechanistic dynamic model and in vivo mechanistic static model approaches on 104 interaction studies. (A) Predicted versus observed AUC ratio with MDM. (B) Predicted versus observed AUC ratio with IMSM. (C) MDM-predicted versus IMSM-predicted AUC ratio. The line is the $y=x$ line.

IMSM:In vivo mechanistic static model; MDM: Mechanistic dynamic model. 


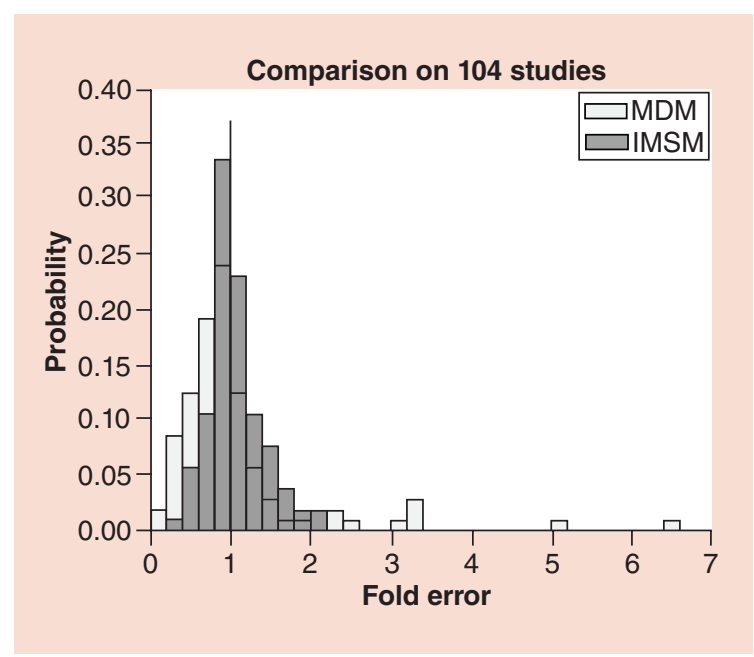

Figure 3. Histogram of the fold errors (= predicted/ observed AUC ratio) for the comparison of mechanistic dynamic model and in vivo mechanistic static model approaches on 104 studies.

The vertical line at $x=1$ represents the ideal value (no prediction bias).

IMSM:In vivo mechanistic static model;

MDM: Mechanistic dynamic model.

scientific background because it combines physiology, pharmacokinetics and mathematics in a comprehensive way. As such, it is a powerful framework, expected to yield accurate predictions. On the other hand, obviously, the success of the PBPK approach depends heavily on the correct specification of most parts of the model. If important pharmacokinetic processes are not taken into account in the model (such as active transport), or some parameters that are erroneous (such as a $\mathrm{Ki}$ ), the predictions may be inaccurate. Hence it may be argued that the performances of the PBPK approach might have been better if the model had been refined for each example considered. The issue of poor prediction with the PBPK approach is not new. Prediction of the PK of a single drug (i.e., not in the context of DDI) from in vitro data with a PBPK model was shown to be inaccurate in many instances [39-41]. The problem is usually solved either by fitting the PBPK model to the clinical data to estimate some parameters [42] or by incorporating scaling factors from animal experiments into the model [39,40]. Although these approaches work quite well: first, they introduce an empirical adjustment, which is in contradiction with the philosophy of the PBPK model; second, they question the ability of the PBPK approach to make quantitative predictions solely from in vitro data.

Owing to its good predictive performances, we suggest that the IMSM approach could be used in the context of drug development during the Phase I of clinical trials. Assume, for example, that in vitro studies have shown that the drug candidate is a substrate of CYP3A4 with a high $\mathrm{fm}$, and an inhibitor of CYP2D6 with low Ki. Then, two prototype clinical DDI studies have to be carried out: one with a strong inhibitor of CYP3A4 (e.g., ketoconazole) and another with a sensitive substrate of CYP2D6 (e.g., dextromethorphan). Since the $\mathrm{IR}_{3 \mathrm{~A} 4}$ of ketoconazole and the $\mathrm{CR}_{2 \mathrm{D} 6}$ of dextromethorphan are already known from previous data, the $\mathrm{CR}_{3 \mathrm{~A} 4}$ and the $\mathrm{IR}_{2 \mathrm{D} 6}$ of the drug candidate can be calculated after completion of these studies by using Equations 8 and 9. Thereafter, the AUC ratio of drug interactions with all potential CYP3A4 inhibitors and CYP2D6 substrates included in the DDI-predictor website may be obtained by using Eguation 6. The computation procedure is very quick, easy and inexpensive. Then, depending on the intended use of the drug candidate and its safety margin, confirmatory studies with selected substrates or inhibitors could be undertaken. As a result, implementation of the IMSM approach could accelerate the assessment of the DDI profile of drug candidate during drug development.

Finally, in the context of drug prescription, the IMSM approach can be useful for anticipating DDIs. This may help to select a drug combination less at risk of strong interaction, or to adjust the dose of substrates with narrow safety margin. Many interactions predicted as strong (AUC ratio >5) have not been studied experimentally $[12-14,17,18]$.

\section{Conclusion}

The in vivo approach coupled with a mechanistic static model (IMSM) has confirmed his good predictive performances on a large panel of 628 DDIs, with $85 \%$ of predictions within 1.5 -fold of the observed value. This approach compared also favourably with the PBPK approach implemented in Simcyp, on a panel of 104 interaction studies. The IMSM approach is a valuable, inexpensive and simple alternative for the prediction of metabolic interactions mediated by cytochromes. It may be of interest for in both drug development and management of DDIs in clinical practice.

\section{Financial \& competing interests disclosure}

The authors have no relevant affiliations or financial involvement with any organization or entity with a financial interest in or financial conflict with the subject matter or materials discussed in the manuscript. This includes employment, consultancies, honoraria, stock ownership or options, expert testimony, grants or patents received or pending, or royalties.

No writing assistance was utilized in the production of this manuscript. 
- The magnitude of a pharmacokinetic interaction is usually expressed as the ratio of the victim drug AUC given in combination with an interacting drug (i.e., inducer or inhibitor) to the victim drug AUC given alone.

- Quantitative prediction of the magnitude of a drug-drug interaction (DDI) is useful to identify the clinical interaction studies to be performed during drug development, and the dosing adaptation to be made in the context of drug prescription.

- The prediction models. Two approaches for quantitative prediction of DDIs mediated by inhibition or induction of cytochromes are the mechanistic dynamic interaction model (MDM) based on in vitro data plugged into a physiologically based pharmacokinetic model, and the mechanistic static interaction model based on in vivo data (IMSM).

- Evaluation of the IMSM predictions. The predictive performances of IMSM (implemented in www.ddipredictor.org) were evaluated on a panel of 628 DDIs. The IMSM yielded $85 \%$ of predictions within $1.5-$ fold of the observed value. The IMSM approach works correctly if cytochromes are the main interaction mechanism, and the kinetics of the substrate is (at least approximately) linear.

- Comparison of the IMSM and MDM predictions. The predictive performances of IMSM and MDM (implemented in Simcyp software) were compared on a set of 104 DDIs. The predictive performances of IMSM were better than those of MDM: median fold error 1 versus 0.86 on 104 studies, $p=0.02$, interquartile fold error 0.40 versus 0.52 .

- The IMSM approach is a quick, inexpensive and simple alternative for the prediction of metabolic interactions mediated by cytochromes. It may be of interest for in both drug development and management of DDIs in clinical practice.

- The MDM approach remains the best approach for the prediction of DDIs involving transporters, provided that the PBPK model is correctly specified.

\section{Ethical conduct of research}

The authors state that they have obtained appropriate institutional review board approval or have followed the principles outlined in the Declaration of Helsinki for all human or animal experimental investigations. In addition, for investigations involving human subjects, informed consent has been obtained from the participants involved.

\section{References}

1 Kiang TK, Ensom MH, Chang TK. UDP-

glucuronosyltransferases and clinical drug-drug interactions. Pharmacol. Therap. 106(1), 97-132 (2005).

2 Backman JT, Kivisto KT, Olkkola KT, Neuvonen PJ. The area under the plasma concentration-time curve for oral midazolam is 400 -fold larger during treatment with itraconazole than with rifampicin. Eur. J. Clin. Pharmacol. 54(1), 53-58 (1998).

3 European Medicines Agency. Guideline on the investigation of drug interactions. Committee for Human Medicinal Products (CHMP) (2012).

4 Food and Drug Administration. Guidance for Industry: Drug Interaction Studies - Study Design, Data Analysis, Implications for Dosing, and Labeling Recommendations. US FDA, MD, USA (2012).

5 Tucker GT. The rational selection of drug interaction studies: implications of recent advances in drug metabolism. Int. J. Clin. Pharmacol. Ther. Toxicol. 30(11), 550-553 (1992).

6 Tucker GT, Houston JB, Huang SM. Optimizing drug development: strategies to assess drug metabolism/ transporter interaction potential - towards a consensus. $\mathrm{Br}$. J. Clin. Pharmacol. 52(1), 107-117 (2001).
7 Blanchard N, Richert L, Coassolo P, Lave T. Qualitative and quantitative assessment of drug-drug interaction potential in man, based on $\mathrm{K}_{\mathrm{i}}, \mathrm{IC}_{50}$ and inhibitor concentration. Curr. Drug Metab. 5(2), 147-156 (2004).

8 Brown HS, Ito K, Galetin A, Houston JB. Prediction of in vivo drug-drug interactions from in vitro data: impact of incorporating parallel pathways of drug elimination and inhibitor absorption rate constant. Br. J. Clin. Pharmacol. 60(5), 508-518 (2005).

9 Rostami-Hodjegan A, Tucker G. 'In silico' simulations to assess the 'in vivo' consequences of 'in vitro' metabolic drug-drug interactions. Drug Discov. Today Technol., 1(4), 441-448 (2004).

10 Rostami-Hodjegan A, Tucker GT. Simulation and prediction of in vivo drug metabolism in human populations from in vitro data. Nat. Rev. Drug Discov. 6(2), 140-148 (2007).

11 Einolf HJ. Comparison of different approaches to predict metabolic drug-drug interactions. Xenobiotica 37(10-11), 1257-1294 (2007).

12 Ohno Y, Hisaka A, Suzuki H. General framework for the quantitative prediction of CYP3A4-mediated oral drug interactions based on the AUC increase by coadministration of standard drugs. Clin. Pharmacokinet. 46(8), 681-696 (2007).

13 Ohno Y, Hisaka A, Ueno M, Suzuki H. General framework for the prediction of oral drug interactions caused by CYP3A4 induction from in vivo information. Clin. Pharmacokinet. 47(10), 669-680 (2008).

14 Tod M, Goutelle S, Clavel-Grabit F, Nicolas G, Charpiat B. Quantitative prediction of cytochrome P450 (CYP) 2D6mediated drug interactions. Clin. Pharmacokinet. 50(8), 519-530 (2011).

15 Tod M, Goutelle S, Gagnieu MC, Genophar II WG. Genotype-based quantitative prediction of drug exposure 
for drugs metabolized by CYP2D6. Clin. Pharmacol. Ther 90(4), 582-587 (2011).

16 Tod M, Nkoud-Mongo C, Gueyffier F. Impact of genetic polymorphism on drug-drug interactions mediated by cytochromes: a general approach. AAPS J. 15(4), 1242-1252 (2013).

17 Castellan AC, Tod M, Gueyffier F et al. Quantitative prediction of the impact of drug interactions and genetic polymorphisms on cytochrome P450 2C9 substrate exposure. Clin. Pharmacokinet. 52(3), 199-209 (2013).

18 Goutelle S, Bourguignon L, Bleyzac N, Berry J, ClavelGrabit F, Tod M. In vivo quantitative prediction of the effect of gene polymorphisms and drug interactions on drug exposure for CYP2C19 substrates. AAPS J. 15(2), 415-426 (2013).

19 Loue C, Tod M. Reliability and extension of quantitative prediction of CYP3A4-mediated drug interactions based on clinical data. AAPS J. 16(6), 1309-1320 (2014).

20 Hisaka A, Ohno Y, Yamamoto T, Suzuki H. Theoretical considerations on quantitative prediction of drug-drug interactions. Drug Metab. Pharmacokinet. 25(1), 48-61 (2010).

21 Rowland M, Tozer TN. Clinical Pharmacokinetics and Pharmacodynamics: Concepts and Application. Lippincott William \& Wilkins, MD, USA (2011).

22 Fahmi OA, Maurer TS, Kish M, Cardenas E, Boldt S, Nettleton D. A combined model for predicting CYP3A4 clinical net drug-drug interaction based on CYP3A4 inhibition, inactivation, and induction determined in vitro. Drug. Metab. Dispos. 36(8), 1698-1708 (2008).

23 Steelandt J, Jean-Bart E, Goutelle S, Tod M. A prediction model of drug exposure in cirrhotic patients according to Child-Pugh classification. Clin. Pharmacokinet. (2015).

24 Fahmi OA, Hurst S, Plowchalk D et al. Comparison of different algorithms for predicting clinical drug-drug interactions, based on the use of CYP3A4 in vitro data: predictions of compounds as precipitants of interaction. Drug. Metab. Dispos. 37(8), 1658-1666 (2009).

25 Guest EJ, Rowland-Yeo K, Rostami-Hodjegan A, Tucker GT, Houston JB, Galetin A. Assessment of algorithms for predicting drug-drug interactions via inhibition mechanisms: comparison of dynamic and static models. $\mathrm{Br}$. J. Clin. Pharmacol. 71(1), 72-87 (2011).

26 Elsby R, Hilgendorf C, Fenner K. Understanding the critical disposition pathways of statins to assess drug-drug interaction risk during drug development: it's not just about OATP1B1. Clin. Pharmacol. Ther.92(5), 584-598 (2012).

27 Hu ZY. Disposition pathway-dependent approach for predicting organic anion-transporting polypeptide-mediated drug-drug interactions. Clin.Pharmacokinet. 52(6), 433-441 (2013).

28 Varma MV, Lai Y, Kimoto E, Goosen TC, El-Kattan AF, Kumar V. Mechanistic modeling to predict the transporterand enzyme-mediated drug-drug interactions of repaglinide. Pharm. Res. 30 (4), 1188-1199 (2013).

29 Varma MV, Lin J, Bi YA et al. Quantitative prediction of repaglinide-rifampicin complex drug interactions using dynamic and static mechanistic models: delineating differential CYP3A4 induction and OATP1B1 inhibition potential of rifampicin. Drug. Metab. Dispos. 41(5), 966-974 (2013).

30 Chen Y, Mao J, Hop CE. Physiologically based pharmacokinetic modeling to predict drug-drug interactions involving inhibitory metabolite: a case study of amiodarone. Drug. Metab. Dispos. 43(2), 182-189 (2015).

31 Guo J, Zhou D, Li Y, Khanh BH. Physiologically based pharmacokinetic modeling to predict complex drug-drug interactions: a case study of AZD2327 and its metabolite, competitive and time-dependent CYP3A inhibitors. Biopharm. Drug Dispos. 36(8), 507-519 (2015).

32 Kirchheiner J, Meineke I, Steinbach N, Meisel C, Roots I, Brockmoller J. Pharmacokinetics of diclofenac and inhibition of cyclooxygenases 1 and 2: no relationship to the CYP2C9 genetic polymorphism in humans. Br. J. Clin. Pharmacol. 55(1), 51-61 (2003).

33 Jaakkola T, Backman JT, Neuvonen M, Niemi M, Neuvonen PJ. Montelukast and zafirlukast do not affect the pharmacokinetics of the CYP2C8 substrate pioglitazone. Eur. J. Clin. Pharmacol. 62(7), 503-509 (2006).

34 Venkatakrishnan K, Obach RS. In vitro-in vivo extrapolation of CYP2D6 inactivation by paroxetine: prediction of nonstationary pharmacokinetics and drug interaction magnitude. Drug. Metab. Dispos. 33(6), 845-852 (2005).

35 Reese MJ, Wurm RM, Muir KT, Generaux GT, St JohnWilliams L, McConn DJ. An in vitro mechanistic study to elucidate the desipramine/bupropion clinical drug-drug interaction. Drug. Metab. Dispos. 36(7), 1198-1201 (2008).

36 Niemi M, Backman JT, Neuvonen M, Neuvonen PJ. Effects of gemfibrozil, itraconazole, and their combination on the pharmacokinetics and pharmacodynamics of repaglinide: potentially hazardous interaction between gemfibrozil and repaglinide. Diabetologia 46(3), 347-351 (2003).

37 Meyer M, Schneckener S, Ludewig B, Kuepfer L, Lippert J. Using expression data for quantification of active processes in physiologically based pharmacokinetic modeling. Drug. Metab. Dispos. 40(5), 892-901 (2012).

38 Johnson TN, Rostami-Hodjegan A, Tucker GT. Prediction of the clearance of eleven drugs and associated variability in neonates, infants and children. Clin. Pharmacokinet. 45(9), 931-956 (2006).

39 Sayama H, Komura H, Kogayu M, Iwaki M. Development of a hybrid physiologically based pharmacokinetic model with drug-specific scaling factors in rat to improve prediction of human pharmacokinetics. J. Pharm. Sci. 102(11), 41934204 (2013).

40 Ito K, Houston JB. Prediction of human drug clearance from in vitro and preclinical data using physiologically based and empirical approaches. Pharm. Res. 22(1), 103-112 (2005).

41 De Buck SS, Mackie CE. Physiologically based approaches towards the prediction of pharmacokinetics: in vitro-in vivo extrapolation. Expert Opin. Drug Metab. Toxicol. 3(6), 865-878 (2007).

42 Peters SA. Evaluation of a generic physiologically based pharmacokinetic model for lineshape analysis. Clin. Pharmacokinet. 47(4), 261-275 (2008). 